\title{
Parafoveal OCT Angiography Features in Diabetic Patients without Clinical Diabetic Retinopathy: A Qualitative and Quantitative Analysis
}

\author{
Mathilde M. Goudot, ${ }^{1}$ Anne Sikorav, ${ }^{1}$ Oudy Semoun, ${ }^{1}$ Alexandra Miere, ${ }^{1}$ Camille Jung, ${ }^{2}$ \\ Blandine Courbebaisse, ${ }^{3}$ Mayer Srour, ${ }^{1}$ Joseph G. Freiha, ${ }^{4}$ and Eric H. Souied ${ }^{1}$ \\ ${ }^{1}$ Department of Ophthalmology, Centre Hospitalier Intercommunal de Creteil, University Paris Est Creteil, Creteil, France \\ ${ }^{2}$ Centre de Recherche Clinique and Centre de Ressources Biologiques, Centre Hospitalier Intercommunal de Creteil, Creteil, France \\ ${ }^{3}$ Department of Diabetology, Centre Hospitalier Intercommunal de Creteil, University Paris Est Creteil, Creteil, France \\ ${ }^{4}$ INOVAD Institution, Paris, France
}

Correspondence should be addressed to Oudy Semoun; oudysemoun@hotmail.com

Received 3 March 2017; Accepted 27 April 2017; Published 29 June 2017

Academic Editor: Ciro Costagliola

Copyright (C) 2017 Mathilde M. Goudot et al. This is an open access article distributed under the Creative Commons Attribution License, which permits unrestricted use, distribution, and reproduction in any medium, provided the original work is properly cited.

\begin{abstract}
Purpose. To evaluate the capacity of OCT angiography (OCTA) for detecting infraclinical lesions in parafoveal capillaries in diabetic patients without diabetic retinopathy (DR). Methods. This prospective observational cross-sectional case-control study analyzed the superficial and deep capillary plexuses (SCP and DCP) on macular OCTA scans $(3 \times 3 \mathrm{~mm})$ centered on the fovea. We compared 22 diabetic patients (34 eyes included) without DR diagnosis on color fundus photographs, with 22 age- and gender-matched nondiabetic controls (40 eyes included). Qualitative analysis concerned morphological ischemic capillary alterations. Quantitative analysis measured foveal avascular zone (FAZ) size, parafoveal capillary density, and enlargement coefficient of FAZ between SCP and DCP. Results. Neither the qualitative nor quantitative parameters were significantly different between both groups. No microaneurysms or venous tortuosity was observed in any of the analyzed images. On the SCP, the mean FAZ area was $0.322 \pm 0.125 \mathrm{~mm}^{2}$ in diabetic patients and $0.285 \pm 0.150 \mathrm{~mm}^{2}$ in controls, $P=0.31$. On the DCP, the mean FAZ area was $0.444 \pm 0.153 \mathrm{~mm}^{2}$ in cases and $0.398 \pm 0.138 \mathrm{~mm}^{2}$ in controls, $P=0.20$. Conclusion. OCTA did not detect infraclinical qualitative or quantitative differences in parafoveal capillaries of diabetic patients without DR in comparison with nondiabetic controls.
\end{abstract}

\section{Introduction}

Diabetic retinopathy (DR) is the leading cause of blindness in the working-age population in developed countries [1]. By 2035, estimates are that 592 million people will be affected by diabetes mellitus [2]. Early detection of its first signs plays a pivotal role in the management of DR, playing an important role in this significant public health issue [3]. Today, fundus slit lamp biomicroscopy and dilated 7-field stereoscopic $30^{\circ}$ color fundus photographs are the current gold standard for DR screening (standard fields are described according to the Early Treatment Diabetic Retinopathy Study (ETDRS)) [4-6]. OCT angiography (OCTA) is a promising and simple test for retinal vascular examination.
Nevertheless, to this date, there is a lack of evidence concerning its capacity to accurately analyze the retinal capillary network or its capacity to detect early DR changes, by comparison to standard screening tests.

OCTA is a recent noninvasive imaging technique that uses motion contrast to create retinal vascular networks images, by comparing the decorrelation signal among sequential OCT B-scans. In 2015, Spaide et al. demonstrated that OCTA could image separately each vascular layer of interest. The morphological retinal layer features in heathy eyes were described by Savastano et al. This type of specific analysis of either the superficial or the deep retinal capillary plexus or the choroidal vascular network is not possible with fundus fluorescence angiography $[7,8]$. 
OCTA has previously been used to describe retinal microvascular lesions in diabetic patients and mainly macular ischemic lesions [9-22]. In diabetic patients without diabetic retinopathy, some studies have put forward a theory concerning infraclinical microvascular changes: the asymmetry and enlargement on the foveal avascular zone (FAZ) area $[15,16,23,24]$ and the presence of capillary nonperfusion areas adjacent to the FAZ $[16,25]$. Because of its noninvasive nature and its rapidity, OCTA may become a good DRscreening tool. To date, only a few studies have evaluated OCTA in diabetic patients with no clinical DR, but no previous study has used color fundus photographs to ensure proper DR status classification (according to the Early Treatment Diabetic Retinopathy Study (ETDRS)) [26].

This study aimed to evaluate if OCTA can detect infraclinical lesions in the parafoveal capillary network of diabetic patients without diabetic retinopathy (DR) on color fundus photographs. The main outcome measure was the presence of significant differences in qualitative and/or quantitative OCTA features in the parafoveal superficial capillary plexus and/or the deep capillary plexus between diabetic patients without DR and age- and gender-matched nondiabetic controls.

\section{Methods}

In this descriptive prospective cross-sectional case-control study, 22 patients with type 1 or type 2 diabetes mellitus and with no clinical diabetic retinopathy (DR) nor diabetic maculopathy were examined. The study was conducted at the Department of Ophthalmology of the Creteil Hospital between March and September 2015. Twenty-two age- and gender-matched healthy control subjects, scheduled for regular visits, were also recruited during this time. Inclusion criteria were patients with type 1 or type 2 diabetes mellitus and the absence of diabetic retinopathy or maculopathy based on multimodal imaging performed on cases and controls by retina specialists (AS, AM, and MMG). The absence of clinical signs of DR was confirmed by two combined examinations: fundus slit lamp examination and counter analysis of $30^{\circ}$ color fundus photographs. A macular spectral domain OCT scan (SD-OCT) established the absence of macular edema. The exclusion criteria were as follows: refractive errors over 6 diopters in spherical equivalent, severe media opacities, macular edema, and other retinal diseases like age-related macular degeneration, hypertensive retinopathy, central serous chorioretinopathy, and vitreoretinal interface, choroid, or optic nerve diseases. Each patient underwent a questionnaire concerning basic demographics, ophthalmological history, and cardiovascular risk factors. We collected the following data: diabetes duration, glycated hemoglobin values, and glomerular filtration rate if available within the last month preceding inclusion. When the results were not available, new blood tests were performed. All patients underwent fundus biomicroscopy with slit lamp and digital color fundus photographs. As the current literature recommendations for DR screening $[6,25,27,28]$, we performed two $30^{\circ}$ photographs per eye under pupillary dilatation: a posterior pole (centered on the macula) and a nasal pole (centered on the optic disc), using the Canon CR-2 Plus Retinal Camera (Canon Company, London, UK). Standard SD-OCT and Enhanced Depth Imaging-OCT (EDI-OCT) were performed using the Spectralis OCT (Heidelberg Engineering, Heidelberg, Germany). We also registered automatic measurements of retinal foveal thickness as well as the manual measurements corresponding to subfoveal choroidal thickness. Choroidal thickness was measured on EDI-OCT scan at the center of the fovea from the posterior face of the retinal pigment epithelium to the choroidal/scleral junction. In accordance with the terms of the Declaration of Helsinki, the local ethics committee in Creteil approved the study. Informed consent was obtained from all subjects prior to the investigation-related procedures.

2.1. OCT Angiography (OCTA). OCTA scans were acquired using a commercially available RTVue XR Avanti with the AngioVue OCTA software (Optovue Inc., Fremont, CA, USA). The scanning area was captured in $3 \times 3 \mathrm{~mm}$ sections, automatically centered on the fovea. The split-spectrum amplitude decorrelation angiography (SSADA) algorithm [29] revealed the blood flow areas by the decorrelation of two cross-sectional 3D raster scans in order to improve the signal-to-noise ratio and minimize motion artifacts. Each 3dimensional volume was obtained from 2 horizontal and 2 vertical fast B-scan acquisitions, composed of 216 B-scans and acquired in 3.4 seconds each. Each B-scan was composed of $304 \times 304 \mathrm{~A}$-scans acquired in a rate of 70,000 A-scans per second, using a scan beam centered on $840 \mathrm{~nm}$, with a bandwidth of $45 \mathrm{~nm}$. To improve the resolution of images, acquisitions could be repeated during the examination. Motion Correction Technology $\left(\mathrm{MCT}^{\mathrm{TM}}\right)$ reduced motion artifacts, and the software ReVue reduced axial and transversal saccadic motion artifacts. To analyze the retinal vascular network, we used the automated segmentation of the superficial and the deep retinal capillary plexuses (SCP and DCP) included in the RTVue XR Avanti, as previously described $[7,22]$. The preset settings were manually adjusted if automated segmentation errors (due to lack of fixation or cooperation) were present.

2.2. Qualitative and Quantitative Analysis. Three retina specialists examined OCTA scans for the qualitative analysis of parafoveal microvasculature. All patients' and controls' $3 \times 3 \mathrm{~mm}$ OCTA scans were therefore arranged on a slide show to enable a magnified analysis. Thesuccession of images was randomly determined in order to avoid letting the readers know the diabetic status of patients. Each OCTA scan was evaluated next to the corresponding "en face" acquisition in order to distinguish vitreous condensations, motion or position artifacts from capillary loss. We retained the following abnormalities, commonly concordant with DR in literature $[11,12,14,16-18,24,30,31]$ : microaneurysms, venous tortuosity [16], irregular outline, and dropout of the anastomotic vascular arcade of the fovea avascular zone (FAZ) $[18,20,21]$. On the parafoveal capillary network, we noticed capillary loss and modified capillary shapes, that is, dilated capillaries, capillary loops [14, 32, 33], and blind-end capillaries [15]. Capillary loss were defined as nonperfusion 


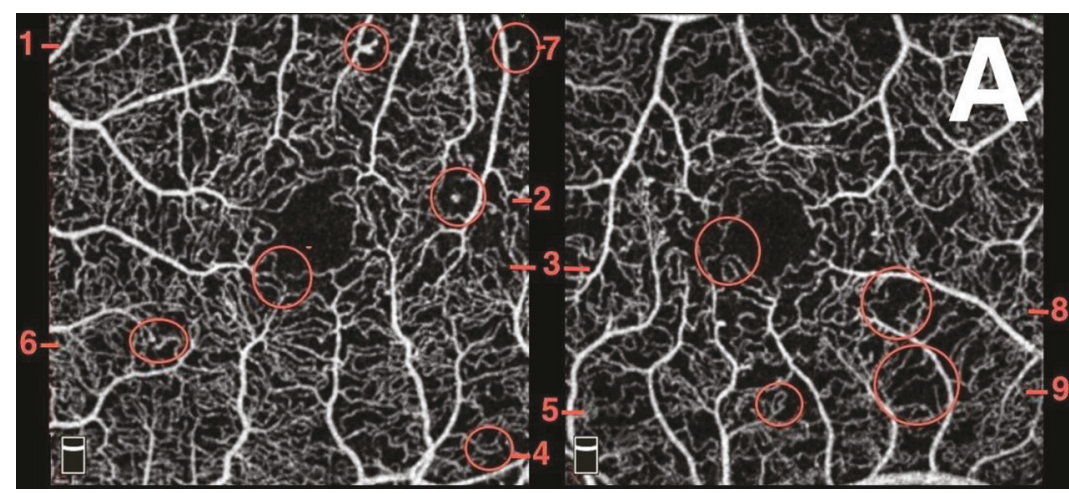

(a)

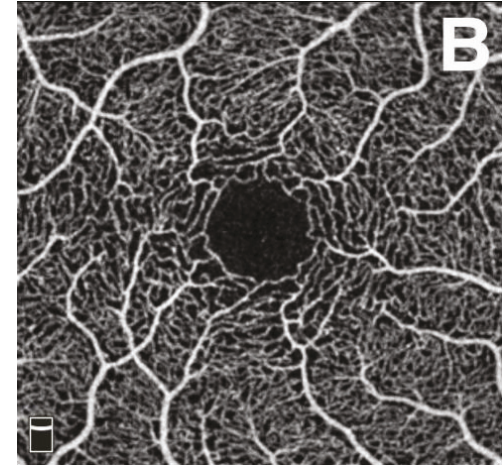

(b)

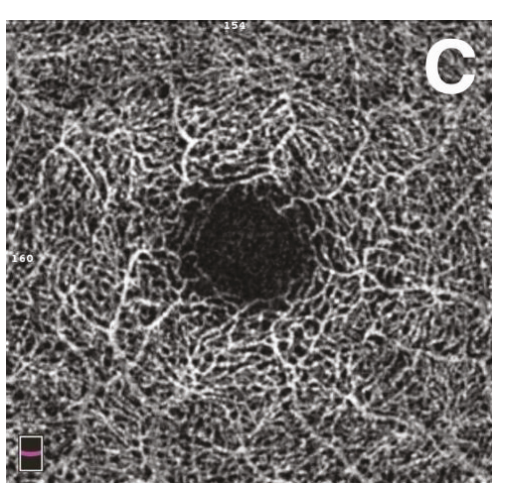

(c)

FIGURE 1: Reference OCT angiography (OCTA) scans for readers for the qualitative analysis of the capillary network. Typical abnormalities in a patient diagnosed with mild nonproliferative diabetic retinopathy on color fundus photographs (a): microaneurysms surrounded by capillary nonperfusion area $(1,2)$, punched out foveal avascular zone (FAZ) outline (3), capillary loops surrounded by capillary nonperfusion area $(4,5)$, blind-end capillaries $(6,7)$, and capillary nonperfusion area $(3,9)$. The images (b) and (c) are reference OCTA scans of a 24-year-old healthy control in the superficial capillary plexus (SCP) (b) and the deep capillary plexus (DCP) (c). In the SCP (b), the FAZ outline is clear and uninterrupted and the capillary plexus is regular, without nonperfusion area. In the DCP (c), the FAZ outline is less clear but still uniform and symmetrical, and no capillary loss appears.

areas characterized by a dark zone without any flow signal, with loss of speckled hyperreflectance homogeneity [21], surrounded by large retinal vessels $[11,34]$ and not compatible with artifacts. A microaneurysm was defined as a round, saccular, or fusiform focal hypersignal [17]. A blind-end capillary is a dilated interrupted terminal vessel surrounded by an area of capillary loss [15]. Typical images of these abnormalities and a normal OCTA scan of each plexus were given to readers as a reference before the analysis (Figure 1).

Quantitative analysis was performed by one retina specialist (MMG) with Angioanalytics quantification software of Optovue system. We measured three vascular parameters: FAZ area, parafoveal vascular density, and parafoveal intercapillary area. The "nonflow area" tool automatically defines borders of FAZ and measures its area (Figures 2(a) and 2(b)). The reader could manually draw the boundaries in case of inaccuracies. The following parameters were calculated: the coefficient of enlargement of FAZ between the SCP and the DCP (resp., sFAZ and dFAZ) denoted $C$. This $C$ value was calculated by the difference between the FAZ area of both plexuses normalized with sFAZ: $C=(\mathrm{dFAZ}-\mathrm{sFAZ}) / \mathrm{sFAZ}$. The vascular density of the parafoveal area was assessed by
2 different methods. With the first method, the "flow area" tool quantified the area of vessels, denoted $A$, in a $1.3 \mathrm{~mm}^{2}$ user-defined region of interest, centered on the fovea (Figures 2(c) and 2(d)).

This selected area corresponded to the juxtafoveal region where capillary losses had been described in previous studies in patients without DR $[16,23]$. The vascular density of the corresponding parafoveal ring, denoted $D$, was then obtained with the formula $D=\mathrm{A} /(1.3-\mathrm{FAZ})$. In this formula, $D=$ vascular density of the parafoveal ring in the central $1.3 \mathrm{~mm}^{2}, \mathrm{FAZ}=$ area of the foveal avascular zone in $\mathrm{mm}^{2}$, and $A=$ capillary flow area of the central $1.3 \mathrm{~mm}^{2}$, as shown in Figure 3. With the second method, the "flow density map" tool assessed the relative density of vascular flow as a percentage of the total area: vascular density was measured in a 1 to $2.5 \mathrm{~mm}$ diameter parafoveal ring (Figures $2(\mathrm{e})$ and 2(f)). Parafoveal intercapillary area (PIA) of the central $1.3 \mathrm{~mm}^{2}$ was calculated by the difference between the parafoveal ring area and vascular area of the "flow" assessment tool with the formula PIA $=1.3-\mathrm{FAZ}-A$, where $\mathrm{FAZ}=$ FAZ area $\left(\mathrm{mm}^{2}\right)$ and $A=$ vascular area of the central $1.3 \mathrm{~mm}^{2}\left(\mathrm{~mm}^{2}\right)$. 


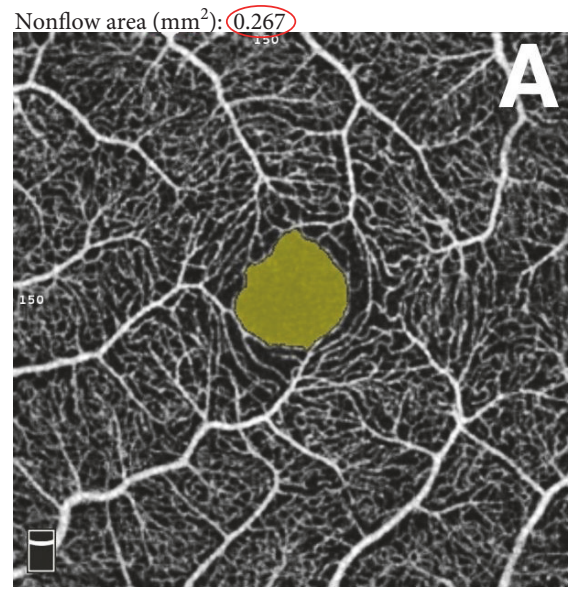

(a)

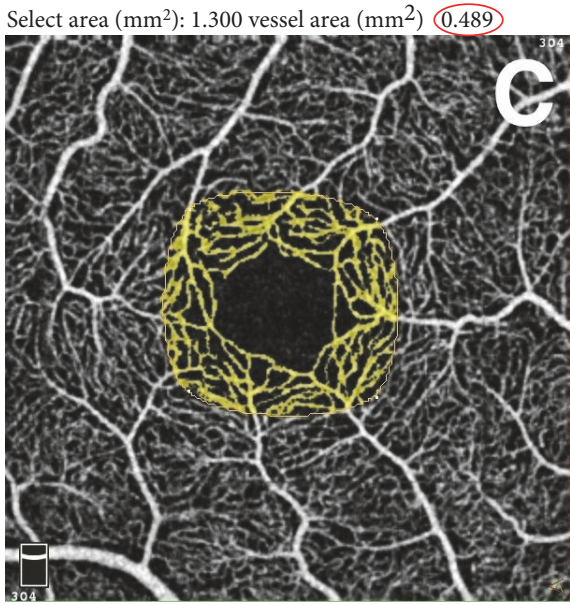

(c)

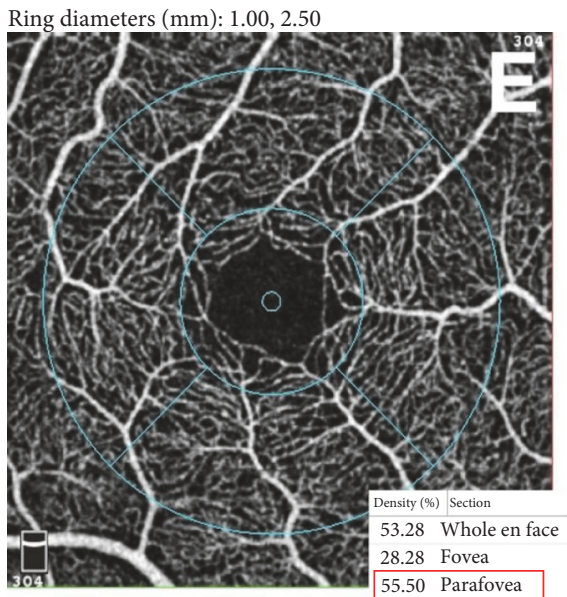

(e)

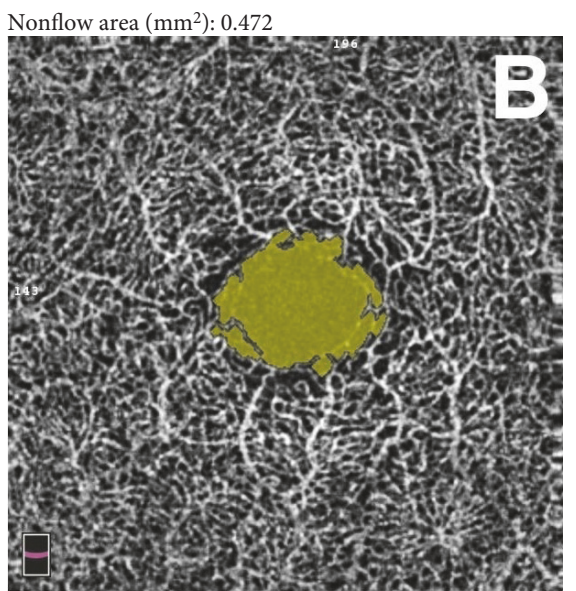

(b)

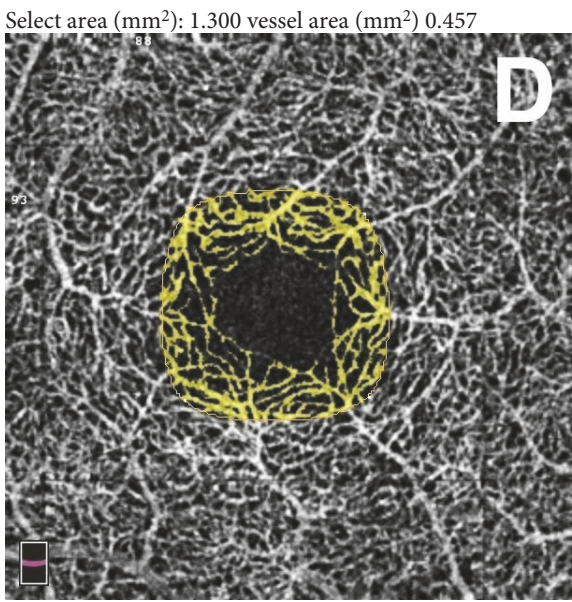

(d)

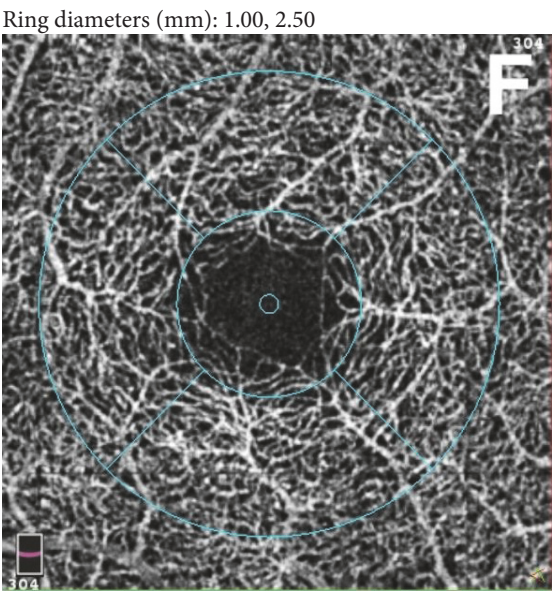

(f)

FIgURE 2: Quantitative analysis of the central retinal capillary network in the superficial capillary plexus (SCP) (left) and the deep capillary plexus (DCP) (right) on OCT angiography scans using the automatized tool of Angioanalytics software on RTVue. The foveal avascular zone area in SCP (a) and DCP (b) was measured using the "nonflow" detection tool. The examiner selected a point in the interest area and the software automatically coloured it in green and measured the area in $\mathrm{mm}^{2}$ (red circle). Parafoveal vascular density was evaluated with 2 different methods (c-f). In the first method, the "flow" detection tool measured the area of vessels in mm ${ }^{2}$ (yellow network, value circled in red) in a central region of interest manually determined at $1.3 \mathrm{~mm}^{2}$ in SCP (c) and DCP (d). In the second method, the "density" tool measured the vascular density in \% in a 1 to $2.5 \mathrm{~mm}$ radius parafoveal ring in SCP (e) and DCP (f). The value framed in red is the mean density of the whole parafoveal ring noticed for our analysis. 


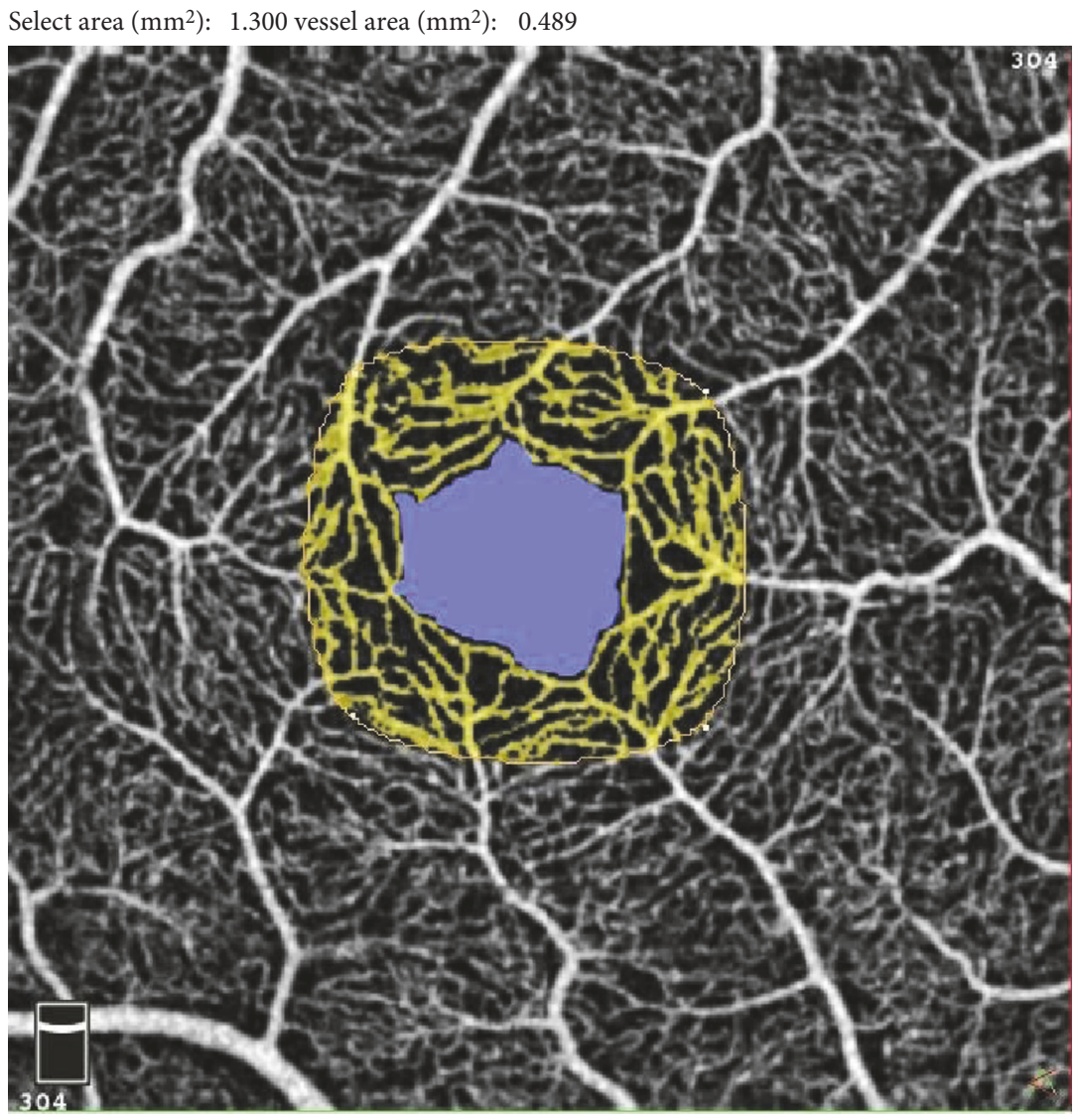

Figure 3: Representation of the first measurement's method of the parafoveal vascular density. The "flow" quantification tool measured the area of vessels in the central $1.3 \mathrm{~mm}^{2}$ user-defined region (yellow). The vascular density in $\%$ in the corresponding parafoveal ring $(D)$ was then obtained with the formula $D=A /(1.3-\mathrm{FAZ})$ with FAZ $\left(\mathrm{mm}^{2}\right)=$ area of the foveal avascular zone (blue) and $A\left(\mathrm{~mm}^{2}\right)=$ vessels' area in the central $1.3 \mathrm{~mm}^{2}$.

2.3. Statistical Analysis. Qualitative variables are described in percentages. Comparisons of qualitative variables were performed using Mac Nemar's $\mathrm{Chi}^{2}$ test. The quantitative analysis compared each case to his age- and gender-matched control. Quantitative variables are described by their mean with standard deviation. Comparisons of means for paired variables were performed using the paired Student $t$-test. $P$ values less than 0.05 were retained as significant. Analysis was performed with STATA version 13.0/SE (StataCorp. 2013. Stata Statistical Software: Release 13, College Station, TX, StataCorp LP). The coefficient regression values were calculated using the linear regression method. Multivariate analyses took into account all of the collected factors associated with the severity of the diabetic retinopathy [35]: age, duration of the diabetes, glycated hemoglobin level, and glomerular filtration rate calculated with the Modification of Diet in Renal Disease study equation (MDRD).

\section{Results}

We examined 22 diabetic patients (15 women and 7 men) without diabetic retinopathy and maculopathy at fundus slit-lamp examination, fundus photographs, and Spectral Domain OCT. Table 1 summarizes the subjects' demographics and vascular risk factors. The mean age of patients was $51 \pm 17$ years, range (16-73). Thirty-two patients were Caucasian, 9 African, 1 Asian, and 2 Hispanic. The same multimodal imaging was performed with the 22 genderand age-matched controls. All patients and controls underwent OCTA that includes a simultaneous acquisition of the corresponding OCT B-scan and OCT "en face." In the analysis, we excluded fourteen eyes (10 diabetics and 4 controls) because of low-quality images. The final anonymized 74 OCTA scans of 34 diabetic and 40 control eyes were randomly disposed on a slide show in order to proceed to qualitative analysis (each slide contained one individual plexus scan). Readers looked for abnormalities blinded to patient status: diabetic or control.

Diabetic patients' clinical and laboratory findings are presented in Table 1 . The diabetic patients' population consisted of $3(14 \%)$ patients with type 1 diabetes and $19(86 \%)$ patients with type 2 diabetes. Six (27\%) patients had been referred at the first diagnosis of diabetes. Diabetic patients presented with an average glycated hemoglobin level of $10.5 \%$, range (6.5-14.4). Seventeen (77\%) patients took oral antidiabetes drugs and $9(41 \%)$ patients used insulin. The mean duration of diabetes was $4.8 \pm 4.1$ years, range (0-13). The control of glycemia was variable, with a mean glycated hemoglobin level of $8.5 \pm 2.9 \%$, range (5.9-17). Besides diabetes, the diabetic group had a higher rate of additional cardiovascular risk 
TABLE 1: Demographics, vascular risk factors, and characteristics of the diabetes.

\begin{tabular}{|c|c|c|c|c|}
\hline & & \multicolumn{2}{|c|}{ Mean \pm SD (range) } & $P^{*}$ \\
\hline \multirow{6}{*}{ Vascular risk factors } & Age: years (matched) & $51 \pm 17(16-73)$ & $51 \pm 17(16-73)$ & 0.24 \\
\hline & Women/men (matched) & $7 / 15$ & $7 / 15$ & - \\
\hline & Hypertension: $N$ & 20 & 12 & 0.05 \\
\hline & Dyslipidemia: $N$ & 10 & 6 & 0.25 \\
\hline & Tobacco: pack years & $5 \pm 2(0-55)$ & $7 \pm 2(0-30)$ & 0.51 \\
\hline & BMI: $\mathrm{kg} / \mathrm{m}^{2}$ & $30 \pm 7(21-50)$ & $24 \pm 5(9-31)$ & 0.0003 \\
\hline \multirow{8}{*}{ Diabetes } & Type of diabetes: $1 / 2$ & $3 / 20$ & - & - \\
\hline & Duration of diabetes: years & $4.8 \pm 4.1(0-13)$ & - & - \\
\hline & Insulin: yes/no & $9 / 13$ & - & - \\
\hline & Oral antidiabetic drugs: yes/no & $17 / 5$ & - & - \\
\hline & $\mathrm{HbA}_{1} \mathrm{c}: \%$ & $8.5 \pm 2.9(5.9-17)$ & - & - \\
\hline & Diabetic nephropathy: yes/no & $2 / 20$ & - & - \\
\hline & MDRD: $\mathrm{mL} / \mathrm{min} / 1.73 \mathrm{~m}^{2}$ & $106 \pm 40(44-185)$ & - & - \\
\hline & Diabetic neuropathy: yes/no & $0 / 22$ & - & - \\
\hline
\end{tabular}

factors than the control population, with a higher prevalence of hypertension $(P=0.05)$ and a higher body mass index $(P<0.001)$. There was no significant difference in other cardiovascular risk factors (tobacco consumption, $P=0.51$; dyslipidemia, $P=0.25$ ). Foveal retinal thickness was significantly thinner in diabetic patients (Table 2, Figure 4(a)): mean foveal retinal thickness was $257 \pm$ $34 \mu \mathrm{m}$ in the diabetic group versus $277 \pm 44 \mu \mathrm{m}$ in the control group, $P<0.01$, CI 95\% $(-34 ;-7)$. Choroidal subfoveal thickness was not significantly different between both groups: $264 \pm 64 \mu \mathrm{m}$ in cases versus $294 \pm 68 \mu \mathrm{m}$ in controls, $P=0.06$, CI $95 \%(-60 ;-1)$, and is concordant with the mean choroidal subfoveal thickness described in the current literature for healthy adult subjects [36].

In the qualitative analysis, no venous tortuosity and no microaneurysm was found in neither plexus for both groups. No difference was noticed either when looking for irregular outlines and punched out borders of the foveal avascular zone (FAZ), as well as nonperfusion areas or morphological capillary network abnormalities (i.e., dilated capillaries, capillary loops, and blind-end capillaries).

The quantitative analysis consisted in a paired Student $t$-test comparing each measured parameter of diabetic eye to his matched control eye. As presented in Table 2, none of the studied vascular parameters was significantly altered in the diabetic group without diabetic retinopathy (DR) by comparison to controls (Figure 4). On both plexuses, the FAZ area was not significantly different between the two groups but its mean was higher in diabetic patients (Figure 4(b)). The superficial FAZ (sFAZ) area measured $0.322 \pm 0.125 \mathrm{~mm}^{2}$ in diabetic patients versus $0.285 \pm$ $0.150 \mathrm{~mm}^{2}$ in controls, with a mean difference of $0.037 \pm$ $0.223 \mathrm{~mm}^{2}$, CI 95\% (-0.036; 0.109), $P=0.31$. The deep FAZ (dFAZ) area measured $0.444 \pm 0.153$ versus $0.398 \pm$ $0.138 \mathrm{~mm}^{2}$ with a mean difference of $0.046 \pm 0.221 \mathrm{~mm}^{2}$, CI 95\% (-0.025; 0.118), $P=0.20$. There was no association between the areas of $s F A Z$ or dFAZ and the age, the glomerular filtration rate, the duration, and the control of diabetes on univariate and multivariate regression analyses. The coefficient of enlargement of FAZ was not significantly different between both groups (Figure 4(c)). The mean coefficient of enlargement was higher in the control group, it was $0.43 \pm 0.32$ in cases and $0.60 \pm 0.61$ in controls, $P=0.14$, CI 95\% (-0.39; 0.06). Parafoveal vascular densities were not significantly different between the two groups in both measuring methods. Their means were lower in the diabetic group except for the parafoveal density in the deep capillary plexus measured with the first method by comparison to controls (Table 2, Figures 4(d) and 4(e)). The "flow" quantification (method 1) measured a lower density than the automatized measurement (method 2): for example, in the superficial capillary plexus with both groups values, the mean density was $49.2 \pm 3.6 \%$ in the diabetic group and $55.1 \pm 2.0 \%$ in controls, $P<0.001$. The parafoveal intercapillary area of the central $1.3 \mathrm{~mm}^{2}$ was not significantly different between the two groups in both plexuses, and its mean was higher in diabetic patients (Table 2, Figure 4(f)).

\section{Discussion}

In the current global context of alarmingly escalating rates in prevalence and incidence of diabetes mellitus [3], efforts should be aimed towards understanding early retinal vascular changes in order to detect early biomarkers. This would allow for more efficient screening of diabetic retinopathy (DR), before complications arise.

The description of DR with OCT angiography is recent, and data in diabetic patients without clinical DR is scarce and not always concordant.

To our knowledge, this is the first study on OCTA using color fundus photographs to ensure proper DR status classification. The ETDRS classification has been established as a reference by comparing FA findings to fundus 


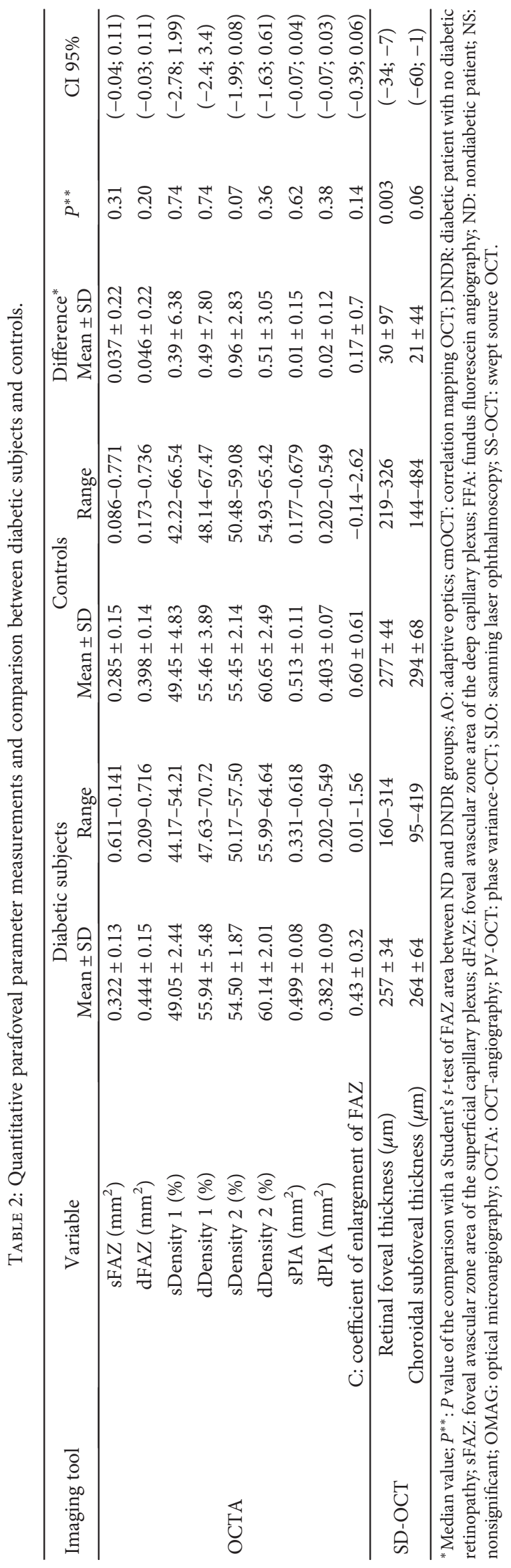




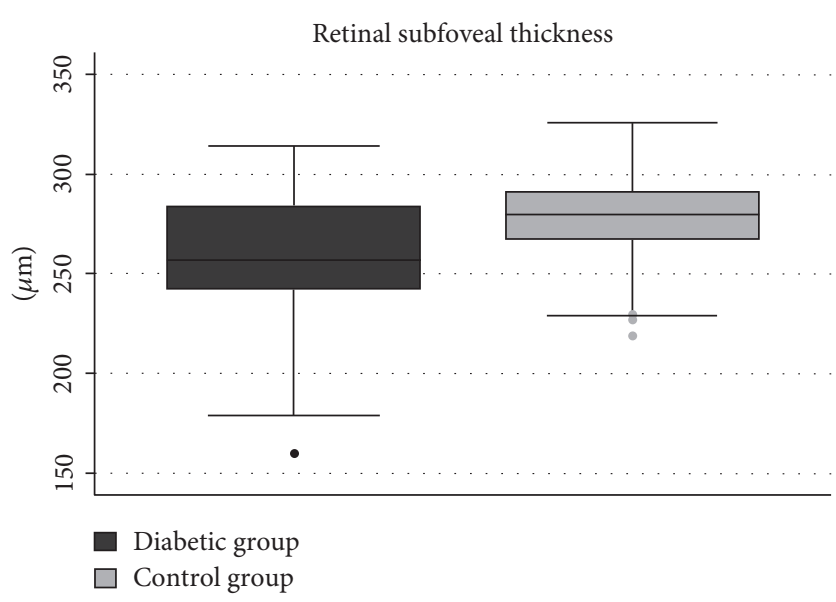

(a)

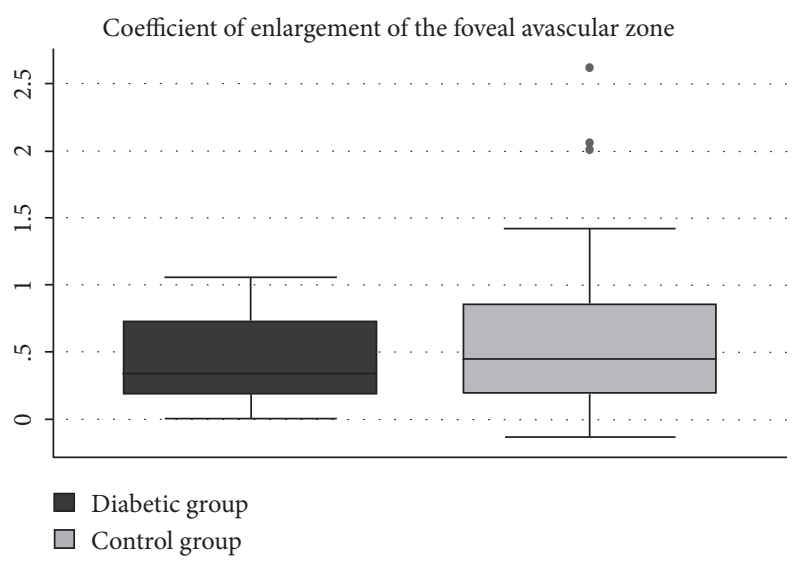

(c)

Vascular density: method 2

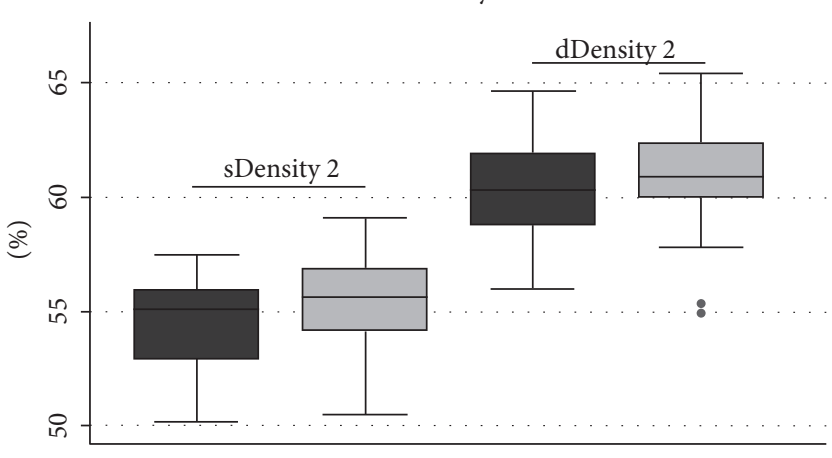

Diabetic group

Control group

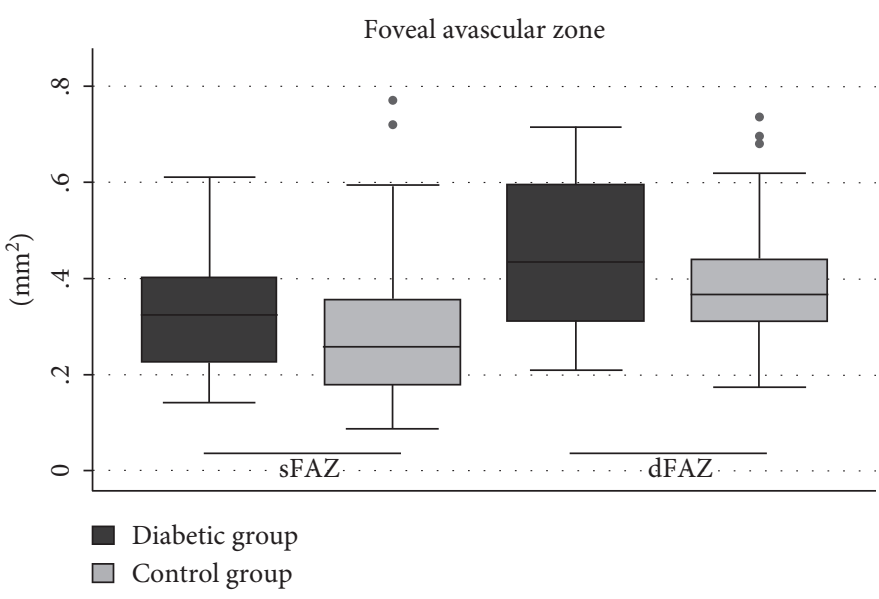

(b)

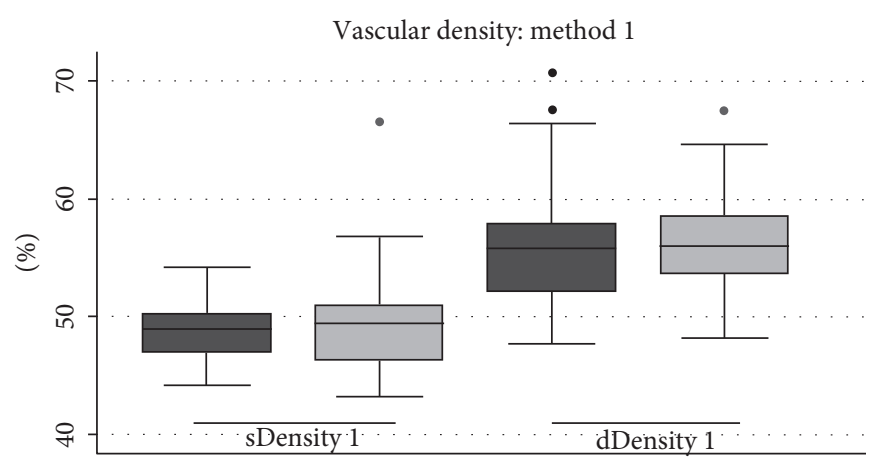

Diabetic group

$\square$ Control group

(d)

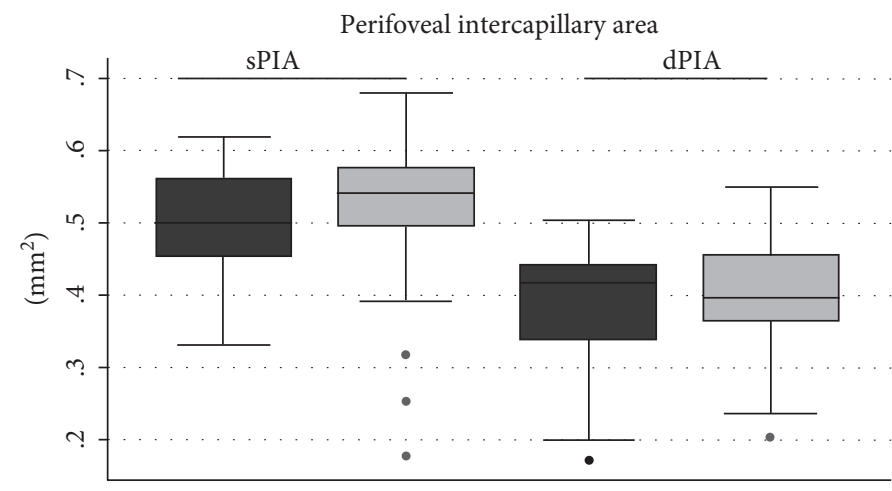

Diabetic group

Control group

(e)

(f)

Figure 4: Comparison and scope of variation of the quantitative parameters of the parafoveal capillary network and the retinal foveal thickness in diabetic patients without DR and their age- and gender-matched controls. None of the quantitative parameters measured was significantly different between the two groups. The foveal avascular zone (FAZ) area in the superficial and the deep capillary plexuses (SCP and DCP) (a), the coefficient of enlargement between FAZ in SCP and DCP (b), the parafoveal vascular density measured with methods 1 (c) and 2 (d), and the parafoveal intercapillary area (PIA) (e) were comparable in both groups. The retinal foveal thickness was significantly thinner in diabetic patients, with a wide scope of variation (f).

photographs [26], and studies have subsequently demonstrated better performance of mydriatic color fundus photographs by comparison to the fundus biomicroscopy for the screening of DR [5, 6, 37]. Furthermore, ophthalmologists often underscore DR in case of mild nonproliferative DR with few microaneurysms and microhemorrhages $[5,37]$. 

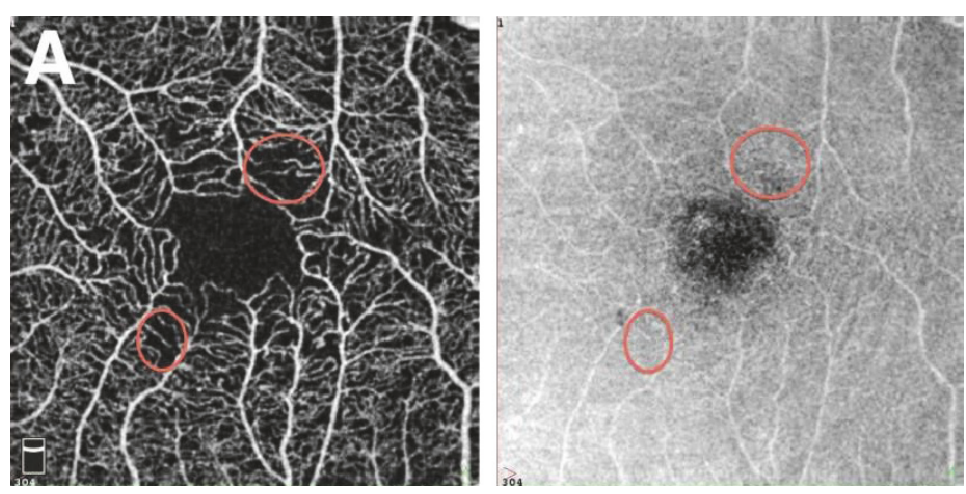

(a)

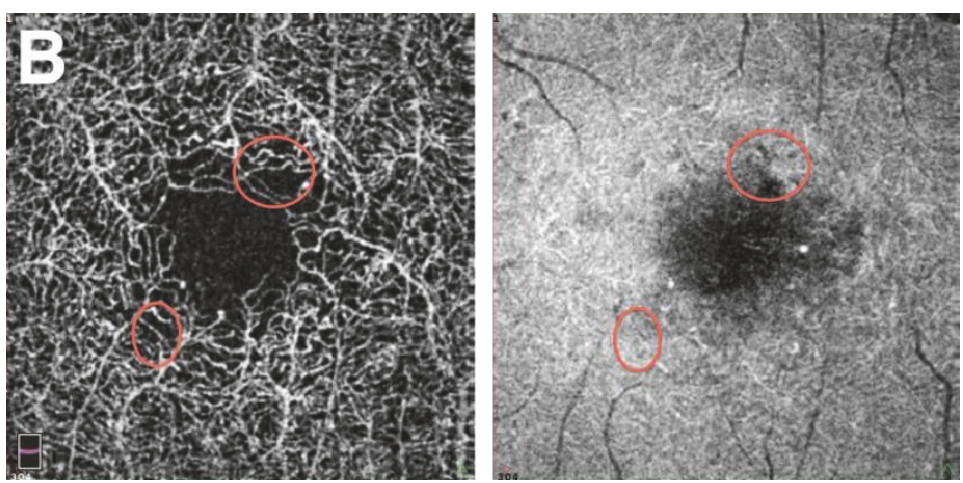

(b)
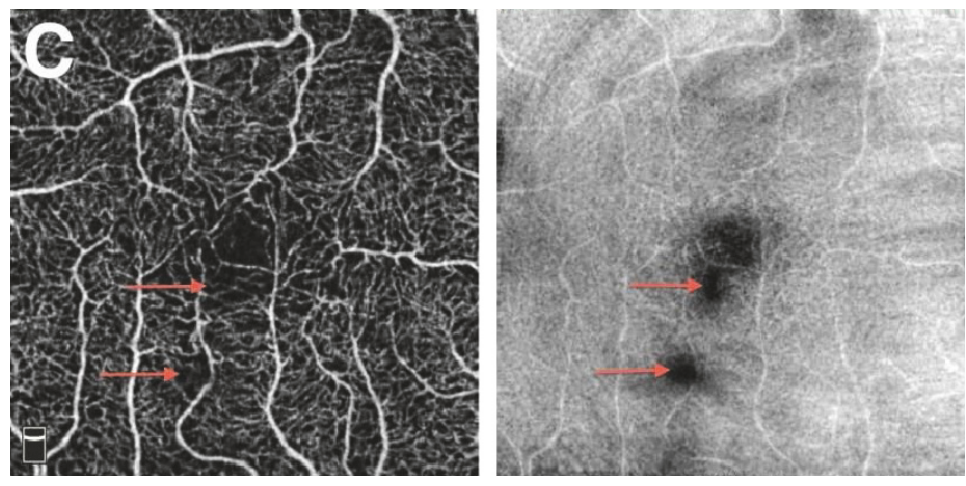

(c)

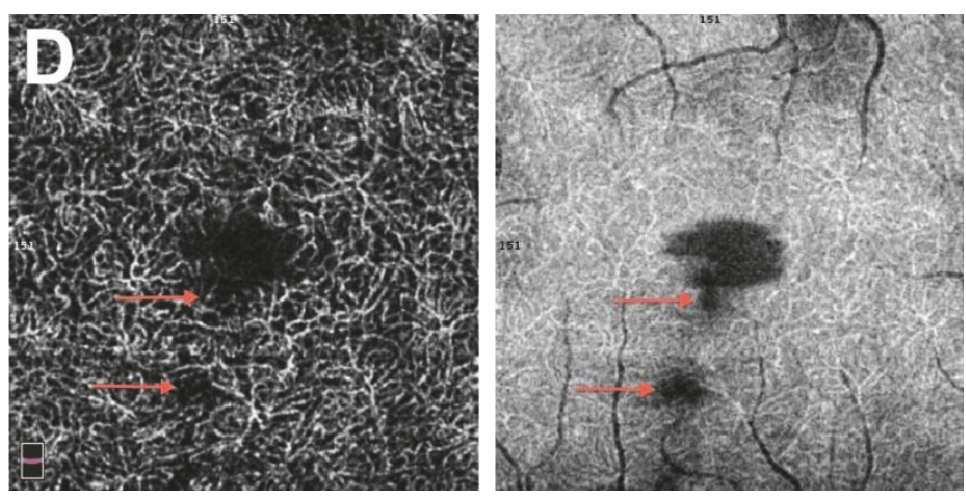

(d)

Figure 5: Continued. 

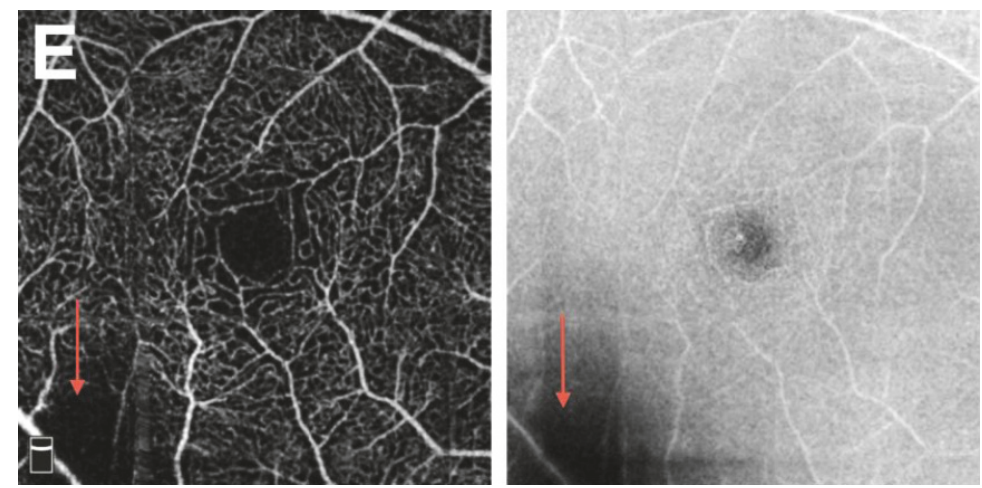

(e)
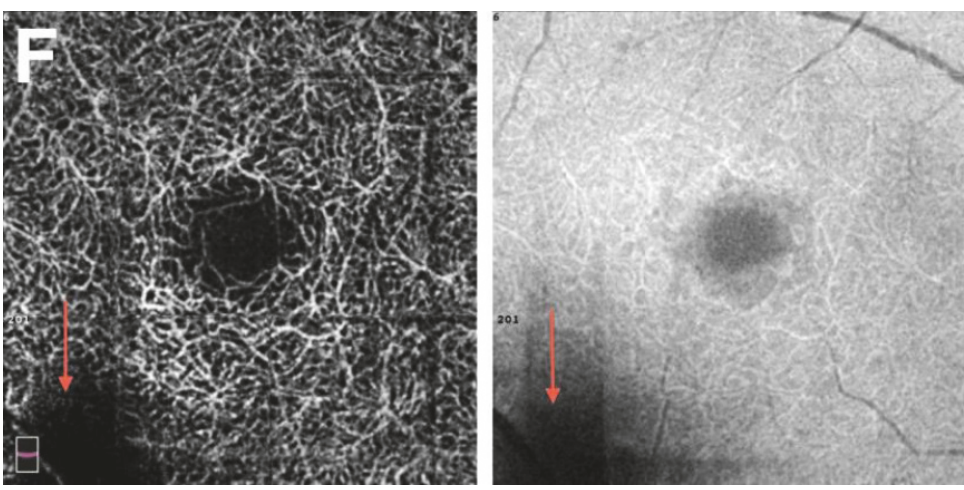

(f)

FIGURE 5: Interest of the "en face" OCT acquisition next to the $3 \times 3 \mathrm{~mm}$ OCT angiography scan for the qualitative analysis to distinguish frequent focal artifactual losses of signal from real capillary nonperfusion areas. Acquisitions are presented successively in the superficial capillary plexus (SCP) (a, c, e) and the deep capillary plexus (DCP) (b, d, f). Real capillary nonperfusion area (a, b) are well marked out with clearly defined peripheral vessels and the surface of the signal's loss is different in both plexuses (red circles) (b). No loss of signal is noticed on the corresponding OCT "en face" acquisition (red circles). We distinguish those from vitreous condensations (c, d), which are responsible for a mask effect on all underlying layers (red arrows). Their borders are hazy, with peripheral blurred vessels and the lack of signal also present with an analogous shape on the "en face" acquisition (d). Position artifacts (e, f) are often located in corners and are also responsible for identical dark areas on both layers and on the "en face" acquisition (red arrows).

We previously defined a consensual description of the researched lesions on OCTA and transmitted images of typical abnormalities as references for readers (Figure 1). As we looked for very slight modifications of the microvasculature, the use of a software correcting artifacts, as well as the definition of relevant criteria for the detection of artifacts, was essential. Indeed, we noticed that the display of the "en face" acquisition was necessary to analyze OCTA scans. This was in order to prevent bias due to the fact that some artifacts resemble capillary loss (Figures 5(a) and 5(b)). Vitreous condensations (Figures 5(c) and 5(d)) and position artifacts (Figures 5(e) and 5(f)) are typical examples of flow signal deletion in the "en face" OCT and in all underlying vascular layers.

Qualitative descriptions of the parafoveal network in diabetic patients without DR in the literature show conflicting results. Some authors describe a clear and regular appearance of the foveal avascular zone (FAZ) borders of the superficial capillary plexus (SCP) as well as uniform perivascular spaces [15]. However, more recent studies describe significant alterations of the SCP in these patients. These alterations are defined with a composite criterion, which includes FAZ border and parafoveal capillary characteristics [16]. Some studies on healthy subjects have pointed that out.

FAZ outlines can often be irregular in the absence of diabetes [38]. Our qualitative analysis did not find any significant difference between both groups, considering irregular and punched out borders of the FAZ, nonperfusion areas, and morphological changes of the capillary network. No microaneurysm or venous tortuosity was found in OCTA scans in our included diabetic population.

Though enlargement of FAZ in DR $[13,15,16,18,23,24]$ has been well documented, data concerning quantitative capillary analyses are still not concordant in diabetic patients without DR [14-16, 23, 24] compared to nondiabetic controls (Table 3). Fundus fluorescence angiography did not find a significant enlargement of the sFAZ but measured a significant enlargement of the parafoveal intercapillary area in the SCP $(P<0.01, N=7)$ [24]. In another study, OCTA observed a significant enlargement of FAZ in both superficial and deep capillary plexus (resp., in literature: $P=0.04, N=53$ and $P<0.01, N=24)[15,23]$. However, this quantitative difference in FAZ area did not correspond to morphological 
TABLE 3: Foveal avascular zone area measurements in healthy subjects and diabetic patients without diabetic retinopathy in the literature.

\begin{tabular}{|c|c|c|c|c|c|c|}
\hline \multirow{2}{*}{$\begin{array}{l}\text { Author } \\
\text { Tam et al. [42] }\end{array}$} & \multirow{2}{*}{$\begin{array}{l}\text { Method } \\
\text { AOSLO }\end{array}$} & \multirow{2}{*}{$\frac{\text { Status }}{\text { ND }}$} & \multirow{2}{*}{$\begin{array}{l}N \\
10\end{array}$} & \multicolumn{2}{|c|}{$\begin{array}{cc}\text { sFAZ } & \text { dFAZ } \\
\text { Mean } \pm \text { SD }\left(\mathrm{mm}^{2}\right)\end{array}$} & \multirow{2}{*}{$\frac{P^{* *}(\mathrm{sFAZ}, \mathrm{dFAZ})}{-}$} \\
\hline & & & & $0.323 \pm 0.107$ & - & \\
\hline Chui et al. [45] & AOSLO & ND & 32 & $0.32 \pm 0.16$ & - & - \\
\hline Popovic et al. [46] & Dual conjugate $\mathrm{AO}$ & ND & 5 & $0.302 \pm 0.100$ & - & - \\
\hline Nelson et al. [47] & Retinal function imager & ND & 37 & $0.125 \pm 0.070$ & - & - \\
\hline Conrath et al. [18] & FFA & $\mathrm{ND}$ & 31 & $0.152 \pm 0.086$ & - & - \\
\hline John et al. [48] & FFA & ND & 31 & $0.275 \pm 0.074$ & - & - \\
\hline Bresnick et al. [20] & FFA & ND & 20 & $0.35^{*}$ & - & - \\
\hline Mansour et al. [31] & FFA & ND & 27 & $0.405 \pm 0.559$ & - & - \\
\hline Dubis et al. [43] & SLO-FFA & ND & 42 & $0.420 \pm 0.250$ & - & - \\
\hline Arend et al. [49] & SLO-FFA & ND & 52 & $0.205 \pm 0.062$ & - & - \\
\hline Kuehlewein et al. [50] & SS-OCT-OMAG & ND & 19 & $0.304 \pm 0.132$ & $0.486 \pm 0.162$ & - \\
\hline Enfield et al. [51] & cmOCT & ND & 37 & $0.125 \pm 0.070$ & - & - \\
\hline Kim et al. [30] & PV-OCT & ND & 2 & 0.167 & - & - \\
\hline Shahlaee et al. [32] & OCTA & ND & 44 & $0.27 \pm 0.101$ & $0.34 \pm 0.116$ & - \\
\hline Carpineto et al. [44] & OCTA & ND & 6 & $0.251 \pm 0.096$ & - & - \\
\hline Samara et al. [38] & OCTA & ND & 67 & $0.266 \pm 0.097$ & $0.495 \pm 0.227$ & - \\
\hline \multirow{2}{*}{ Arend et al. [24] } & \multirow{2}{*}{ SLO-FFA } & $\mathrm{ND}$ & 21 & $0.231 \pm 0.06$ & - & - \\
\hline & & DNDR & 7 & $0.276 \pm 0.08$ & - & NS \\
\hline \multirow{2}{*}{ de Carlo et al. [16] } & \multirow{2}{*}{ OCTA } & ND & 28 & $0.288 \pm 0.136$ & - & \\
\hline & & DNDR & 61 & $0.348 \pm 0.101$ & - & 0.04 \\
\hline \multirow{2}{*}{ Takase et al. [23] } & \multirow{2}{*}{ OCTA } & ND & 19 & $0.25 \pm 0.06$ & $0.38 \pm 0.11$ & $<0.01$ \\
\hline & & DNDR & 24 & $0.37 \pm 0.07$ & $0.54 \pm 0.13$ & $<0.01$ \\
\hline \multirow{2}{*}{ Di et al. [15] } & \multirow{2}{*}{ OCTA } & ND & 85 & $0.36 \pm 0.11$ & & \\
\hline & & DNDR & 53 & $0.40 \pm 0.16$ & & 0.04 \\
\hline \multirow{2}{*}{ Present study } & \multirow{2}{*}{ OCTA } & ND & 23 & $0.294 \pm 0.150$ & $0.348 \pm 0.02$ & 0.31 \\
\hline & & DNDR & 23 & $0.321 \pm 0.120$ & $0.420 \pm 0.02$ & 0.20 \\
\hline
\end{tabular}

*Mean difference between cases' and controls' values; $P^{* *}: P$ values in the comparison between diabetic subjects and their age- and gender-matched nondiabetic controls with a paired Student's $t$-test; CI 95\%: 95\% confidence interval; sDensity1 and dDensity1: parafoveal ring vascular density measured with the "flow" quantification tool (method 1) respectively, on the SCP and DCP; sDensity2 and dDensity2: parafoveal ring vascular density measured with the "density" quantification tool (method 2) respectively, on the SCP and DCP; DCP: deep capillary plexus; FAZ: foveal avascular zone; sFAZ and dFAZ: FAZ area, respectively, on the SCP and DCP; sPIA and dPIA: parafoveal intercapillary area, respectively, on the SCP and DCP; SCP: superficial capillary plexus; SD: standard deviation; SD-OCT: spectral domain-OCT.

changes of the regularity and appearance of FAZ in the concomitant qualitative analysis [15]. Our results did not show any significant enlargement of the FAZ in the superficial or the deep capillary plexus (sFAZ and dFAZ) of diabetic patients without clinical DR, by comparison to healthy controls.

As previous authors suggested predominant and earlier enlargement of FAZ in the deep capillary plexus (DCP) [14] in DR, we decided to look at this hypothesis: was the absolute value of FAZ area a good criterion to detect early DR? Because of its interindividual critical variability in healthy subjects $[18,20,30,38-43]$, we hypothesized that the naive FAZ area could be a confusing factor in FAZ enlargement's measurement. As a consequence, the coefficient of enlargement of the FAZ between the SCP and DCP was established to detect earlier deep vascular changes independently of individual FAZ variations. $\mathrm{AFAZ}$ is normalized with $\mathrm{sFAZ}$ so that each patient becomes its own control. This coefficient was not significantly higher in the diabetic group than in healthy controls $(P=0.14)$. Moreover, its mean value was higher in the control group with a mean of $0.60 \pm 0.61$ in cases and $0.43 \pm$ 0.32 in controls. These results argue against the earlier alteration of deep vascular network in diabetic patients without DR. We noticed that this value was relevant only if sFAZ is not altered, as we observed it in our qualitative analysis.

The development of DR can be seen either in a linear progression or in a stairstep model. This study could lead to hypothesize a stairstep model of the apparition of ischemic lesions on OCTA, concordant with the first microhemorrhages and microaneurysms on color fundus photographs. The literature also describes obvious vascular lesions with OCTA in mild diabetic retinopathy $[14,15,17,18]$ arguing in favor of the high sensitivity of OCTA to detect early signs of clinical DR.

The interpretation of our results should take into account that the area of the retina analyzed with $3 \times 3 \mathrm{~mm}$ OCTA scans is limited and that the precise correlation of macular ischemia and peripheral DR manifestations remains to be established. Further studies using wider field OCTA, with 
peripheral fundus photographs are necessary to evaluate this correlation. In our study, a single examiner (MMG) performed FAZ measurements using an automatic program imbedded in the software of the OCTA. Literature argues in favor of the reproducibility and repeatability of FAZ measurement with the same method [44]. The mean sFAZ and dFAZ area measured matched with the values of the literature (Table 3).

A noticeable characteristic of our patients is the short duration of diabetes $(4.8 \pm 4.0$ years), with 6 patients examined at the discovery of the disease. Though it allows us to study the very beginning of the disease, we cannot exclude that capillary microchanges existing before clinical DR could not be described because of this relative short duration of diabetes.

In conclusion, OCTA did not detect infraclinical quantitative or qualitative differences in parafoveal capillaries of diabetic patients without DR in comparison with controls. This result suggests that color fundus photographs and OCTA could concord to assess the absence of DR. The increasing distribution of OCTA in the clinical ophthalmological practice and the development of full-field OCTA fundus camera should be able to strengthen these findings on a wider scale. Considering that it is relatively easy to perform, inexpensive, and noninvasive, OCTA could become an efficient screening tool and might create a breakthrough in the solving of one of the major eye health problems in developed countries.

\section{Abbreviations}

AOSLO: Adaptive optics scanning laser ophthalmoscopy

BMI: $\quad$ Body mass index

CI: $\quad$ Confidence interval

cmOCT: Correlation mapping OCT

DCP: $\quad$ Deep capillary plexus

sDensity1 Parafoveal ring vascular density measured with and the "flow" quantification tool (method 1),

dDensity1: respectively, on the superficial and the deep capillary plexus

sDensity2 Parafoveal ring vascular density measured with and the automatized "density" quantification tool

dDensity2: (method 2), respectively, on the superficial and the deep capillary plexus

DNDR: Diabetic patients with no diabetic retinopathy

DR: Diabetic retinopathy

EDI: $\quad$ Enhanced depth imaging

ETDRS: Early treatment diabetic retinopathy study

FAZ: $\quad$ Foveal avascular zone

sFAZ: $\quad$ FAZ area of the superficial capillary plexus

dFAZ: $\quad$ FAZ area of the deep capillary plexus

FFA: $\quad$ Fundus fluorescence angiography

$\mathrm{HbA}_{1} \mathrm{c}$ : Glycated hemoglobin

MDRD: Glomerular filtration rate calculated with the modification of diet in renal disease study equation

ND: $\quad$ Nondiabetic patients

NS: $\quad$ Nonsignificant

OMAG: Optical microangiography
OCT: Optical coherence tomography

OCTA: OCT angiography

PIA: $\quad$ Parafoveal intercapillary area

sPIA Parafoveal intercapillary area, respectively, on

and dPIA: the superficial and the deep capillary plexus

PV- OCT: Phase variance-OCT

SCP: $\quad$ Superficial capillary plexus

SD: $\quad$ Standard deviation

SD-OCT: Spectral domain OCT

SLO: $\quad$ Scanning laser ophthalmoscopy

SS-OCT: $\quad$ Swept source-OCT

SSADA: Split-spectrum amplitude decorrelation angiography

AS: $\quad$ Anne Sikorav

AM: $\quad$ Alexandra Miere

MMG: $\quad$ Mathilde M. Goudot.

\section{Conflicts of Interest}

The authors have no financial disclosures.

\section{Authors' Contributions}

Mathilde M. Goudot, Anne Sikorav, and Oudy Semoun were responsible for the design and conduct of the study. Mathilde M. Goudot, Anne Sikorav, Oudy Semoun, Alexandra Miere, Mayer Srour, Camille Jung, and Blandine Courbebaisse helped in the collection, management, and analysis. Mathilde M. Goudot, Anne Sikorav, Alexandra Miere, Mayer Srour, Camille Jung, and Eric H. Souied contributed to the interpretation of the data. Mathilde M. Goudot, Anne Sikorav, Oudy Semoun, Alexandra Miere, and Eric H. Souied contributed to the preparation, review, or approval of the study.

\section{Acknowledgments}

The authors would like to thank the whole care team of the Department of Ophthalmology of Creteil Hospital for the achievement of this study.

\section{References}

[1] N. Cheung, P. Mitchell, and T. Y. Wong, "Diabetic retinopathy," Lancet, vol. 376, no. 9735, pp. 124-136, 2010.

[2] International Diabetes Federation, IDF Diabetes Atlas, p. 7, International Diabetes Federation, Brussels, Belgium, 6 edition, 2013.

[3] L. S. Geiss, J. Wang, Y. J. Cheng et al., "Prevalence and incidence trends for diagnosed diabetes among adults aged 20 to 79 years, United States, 1980-2012," Jama, vol. 312, no. 12, p. 1218, 2014.

[4] R. Taylor, L. Lovelock, W. M. Tunbridge et al., "Comparison of non-mydriatic retinal photography with ophthalmoscopy in 2159 patients: mobile retinal camera study," BMJ, vol. 301, no. 6763, pp. 1243-1247, 1990.

[5] J. A. Pugh, J. M. Jacobson, W. A. Van Heuven et al., "Screening for diabetic retinopathy. The wide-angle retinal camera," Diabetes Care, vol. 16, no. 6, pp. 889-895, 1993. 
[6] D. Y. Lin, M. S. Blumenkranz, R. J. Brothers, and D. M. Grosvenor, "The sensitivity and specificity of single-field nonmydriatic monochromatic digital fundus photography with remote image interpretation for diabetic retinopathy screening: a comparison with ophthalmoscopy and standardized mydriatic color photography," American Journal of Ophthalmology, vol. 134, no. 2, pp. 204-213, 2002.

[7] R. F. Spaide, J. M. Klancnik, and M. J. Cooney, "Retinal vascular layers imaged by fluorescein angiography and optical coherence tomography angiography," JAMA Ophthalmology, vol. 133, no. 1, p. 45, 2015.

[8] M. C. Savastano, B. Lumbroso, and M. Rispoli, "In vivo characterization of retinal vascularization morphology using optical coherence tomography angiography," Retina, vol. 35, no. 11, pp. 2196-2203, 2015.

[9] M. Zhang, J. Wang, A. D. Pechauer et al., "Advanced image processing for optical coherence tomographic angiography of macular diseases," Biomedical Optics Express, vol. 6, no. 12, p. 4661, 2015.

[10] Y. Jia, S. T. Bailey, T. S. Hwang et al., "Quantitative optical coherence tomography angiography of vascular abnormalities in the living human eye," Proceedings of the National Academy of Sciences, vol. 112, no. 18, pp. E2395-E2402, 2015.

[11] A. Ishibazawa, T. Nagaoka, A. Takahashi et al., "Optical coherence tomography angiography in diabetic retinopathy: a prospective pilot study," American Journal of Ophthalmology, vol. 160, no. 1, pp. 35-44, 2015, e1.

[12] T. S. Hwang, Y. Jia, S. S. Gao et al., "Optical coherence tomography angiography features of diabetic retinopathy," Retina, vol. 35, no. 11, pp. 2371-2376, 2015.

[13] T. S. Hwang, S. S. Gao, L. Liu et al., "Automated quantification of capillary Nonperfusion using optical coherence tomography angiography in diabetic retinopathy," JAMA Ophthalmology, vol. 134, no. 4, pp. 367-373, 2016.

[14] F. J. Freiberg, M. Pfau, J. Wons, M. A. Wirth, M. D. Becker, and S. Michels, "Optical coherence tomography angiography of the foveal avascular zone in diabetic retinopathy," Graefe's Archive for Clinical and Experimental Ophthalmology, vol. 254, no. 6, pp. 1051-1058, 2016.

[15] G. Di, Y. Weihong, Z. Xiao et al., "A morphological study of the foveal avascular zone in patients with diabetes mellitus using optical coherence tomography angiography," Graefe's Archive for Clinical and Experimental Ophthalmology, vol. 254, no. 5, pp. 873-879, 2016.

[16] T. E. de Carlo, A. T. Chin, M. A. Bonini Filho et al., "Detection of microvascular changes in eyes of patients with diabetes but not clinical diabetic retinopathy using optical coherence tomography angiography," Retina, vol. 35 , no. 11 , pp. 2364-2370, 2015.

[17] A. Couturier, V. Mané, S. Bonnin et al., "Capillary plexus anomalies in diabetic retinopathy on optical coherence tomography angiography," Retina, vol. 35, no. 11, pp. 2384-2391, 2015.

[18] J. Conrath, R. Giorgi, D. Raccah, and B. Ridings, "Foveal avascular zone in diabetic retinopathy: quantitative vs qualitative assessment," Eye, vol. 19, no. 3, pp. 322-326, 2005.

[19] E. D. Cole, E. A. Novais, R. N. Louzada, and N. K. Waheed, "Contemporary retinal imaging techniques in diabetic retinopathy: a review," Clinical \& Experimental Ophthalmology, vol. 44, no. 4, pp. 289-299, 2016.

[20] G. H. Bresnick, R. Condit, S. Syrjala, M. Palta, A. Groo, and K. Korth, "Abnormalities of the foveal avascular zone in diabetic retinopathy," Archives of Ophthalmology, vol. 102, no. 9, pp. 1286-1293, 1984.

[21] P. D. Bradley, D. A. Sim, P. A. Keane et al., "The evaluation of diabetic macular ischemia using optical coherence tomography angiography," Investigative Ophthalmology \& Visual Science, vol. 57, no. 2, p. 626, 2016.

[22] S. A. Agemy, N. K. Scripsema, C. M. Shah et al., "Retinal vascular perfusion density mapping using optical coherence tomography angiography in normals and diabetic retinopathy patients," Retina, vol. 35, no. 11, pp. $2353-$ 2363, 2015.

[23] N. Takase, M. Nozaki, A. Kato, H. Ozeki, M. Yoshida, and Y. Ogura, "Enlargement of foveal avascular zone in diabetic eyes evaluated by en face optical coherence tomography angiography," Retina, vol. 35, no. 11, pp. 2377-2383, 2015.

[24] O. Arend, S. Wolf, F. Jung et al., "Retinal microcirculation in patients with diabetes mellitus: dynamic and morphological analysis of perifoveal capillary network," The British Journal of Ophthalmology, vol. 75, no. 9, pp. 514-518, 1991.

[25] M. C. Boucher, J. A. Gresset, K. Angioi, and S. Olivier, "Effectiveness and safety of screening for diabetic retinopathy with two nonmydriatic digital images compared with the seven standard stereoscopic photographic fields," Canadian Journal of Ophthalmology/Journal Canadien d'Ophtalmologie, vol. 38, no. 7, pp. 557-568, 2003.

[26] Classification of diabetic retinopathy from fluorescein angiograms. ETDRS report number 11. Early treatment diabetic retinopathy study research group," Ophthalmology, vol. 98, 5 Supplement, pp. 807-822, 1991.

[27] S. E. Bursell, J. D. Cavallerano, A. A. Cavallerano et al., "Stereo nonmydriatic digital-video color retinal imaging compared with early treatment diabetic retinopathy study seven standard field 35-mm stereo color photos for determining level of diabetic retinopathy," Ophthalmology, vol. 108, no. 3, pp. 572-585, 2001.

[28] G. H. Bresnick, D. B. Mukamel, J. C. Dickinson, and D. R. Cole, "A screening approach to the surveillance of patients with diabetes for the presence of vision-threatening retinopathy," Ophthalmology, vol. 107, no. 1, pp. 19-24, 2000.

[29] Y. Jia, O. Tan, J. Tokayer et al., "Split-spectrum amplitudedecorrelation angiography with optical coherence tomography," Optics Express, vol. 20, no. 4, p. 4710, 2012.

[30] D. Y. Kim, J. Fingler, R. J. Zawadzki et al., "Noninvasive imaging of the foveal avascular zone with high-speed, phase-variance optical coherence tomography," Investigative Ophthalmology \& Visual Science, vol. 53, no. 1, p. 85, 2012.

[31] A. M. Mansour, A. Schachat, G. Bodiford, and R. Haymond, "Foveal avascular zone in diabetes mellitus," Retina, vol. 13, no. 2, pp. 125-128, 1993.

[32] A. Shahlaee, M. Pefkianaki, J. Hsu, and A. C. Ho, "Measurement of foveal avascular zone dimensions and its reliability in healthy eyes using optical coherence tomography angiography," American Journal of Ophthalmology, vol. 161, no. 1, pp. 50-55, 2016, e1.

[33] B. Sander, M. Larsen, C. Engler, H. Lund-Andersen, and H. H. Parving, "Early changes in diabetic retinopathy: capillary loss and blood-retina barrier permeability in relation to metabolic control," ActaOphthalmologica, vol.72, no.5,pp.553-559, 1994.

[34] M. M. Wessel, N. Nair, G. D. Aaker, J. R. Ehrlich, D. J. D'Amico, and S. Kiss, "Peripheral retinal ischaemia, as evaluated by ultra-widefield fluorescein angiography, is associated 
with diabetic macular oedema," The British Journal of Ophthalmology, vol. 96, no. 5, pp. 694-698, 2012.

[35] D. A. Antonetti, R. Klein, and T. W. Gardner, "Diabetic retinopathy," The New England Journal of Medicine, vol. 366, no. 13, pp. 1227-1239, 2012.

[36] V. Manjunath, M. Taha, J. G. Fujimoto, and J. S. Duker, "Choroidal thickness in normal eyes measured using cirrus HD optical coherence tomography," American Journal of Ophthalmology, vol. 150, no. 3, pp. 325-329, 2010, e1.

[37] A. Hutchinson, A. McIntosh, J. Peters et al., "Effectiveness of screening and monitoring tests for diabetic retinopathy - a systematic review," Diabetic Medicine, vol. 17, no. 7, pp. 495-506, 2000.

[38] W. A. Samara, E. A. T. Say, K. CTL et al., "Correlation of foveal avascular zone size with foveal morphology in normal eyes using optical coherence tomography angiography," Retina, vol. 35, no. 11, pp. 2188-2195, 2015.

[39] L. Z. Wu, Z. S. Huang, D. Z. Wu, and E. Chan, "Characteristics of the capillary-free zone in the normal human macula," Japanese Journal of Ophthalmology, vol. 29, no. 4, pp. 406411, 1985.

[40] Y. Zheng, J. S. Gandhi, A. N. Stangos, C. Campa, D. M. Broadbent, and S. P. Harding, "Automated segmentation of foveal avascular zone in fundus fluorescein angiography," Investigative Ophthalmology \& Visual Science, vol. 51, no. 7, p. 3653, 2010.

[41] L. Laatikainen and J. Larinkari, "Capillary-free area of the fovea with advancing age," Investigative Ophthalmology \& Visual Science, vol. 16, no. 12, pp. 1154-1157, 1977.

[42] J. Tam, J. A. Martin, and A. Roorda, "Noninvasive visualization and analysis of Parafoveal capillaries in humans," Investigative Ophthalmology \& Visual Science, vol. 51, no. 3, p. 1691, 2010.

[43] A. M. Dubis, B. R. Hansen, R. F. Cooper, J. Beringer, A. Dubra, and J. Carroll, "Relationship between the foveal avascular zone and foveal pit morphology," Investigative Ophthalmology \& Visual Science, vol. 53, no. 3, pp. 1628-1636, 2012.

[44] P. Carpineto, R. Mastropasqua, G. Marchini, L. Toto, M. Di Nicola, and L. Di Antonio, "Reproducibility and repeatability of foveal avascular zone measurements in healthy subjects by optical coherence tomography angiography," The British Journal of Ophthalmology, vol. 100, no. 5, pp. 671-676, 2016.

[45] T. Y. P. Chui, Z. Zhong, H. Song, and S. A. Burns, "Foveal avascular zone and its relationship to foveal pit shape," Optometry and Vision Science, vol. 89, no. 5, pp. 602-610, 2012.

[46] Z. Popovic, P. Knutsson, J. Thaung, M. Owner-Petersen, and J. Sjöstrand, "Noninvasive imaging of human foveal capillary network using dual-conjugate adaptive optics," Investigative Ophthalmology \& Visual Science, vol. 52, no. 5, pp. 26492655, 2011.

[47] D. A. Nelson, Z. Burgansky-Eliash, H. Barash et al., "Highresolution wide-field imaging of perfused capillaries without the use of contrast agent," Clinical ophthalmology, vol. 5, no. 8, pp. 1095-1106, 2011.

[48] D. John, T. Kuriakose, S. Devasahayam, and A. Braganza, "Dimensions of the foveal avascular zone using the Heidelberg retinal angiogram-2 in normal eyes," Indian Journal of Ophthalmology, vol. 59, no. 1, pp. 9-11, 2011.

[49] O. Arend, S. Wolf, A. Harris, and M. Reim, "The relationship of macular microcirculation to visual acuity in diabetic patients," Archives of Ophthalmology, vol. 113, no. 5, pp. 610-614, 1995.

[50] L. Kuehlewein, T. C. Tepelus, L. An, M. K. Durbin, S. Srinivas, and S. R. Sadda, "Noninvasive visualization and analysis of the human Parafoveal capillary network using swept source OCT optical microangiography," Investigative Ophthalmology \& Visual Science, vol. 56, no. 6, pp. 3984-3988, 2015.

[51] J. Enfield, E. Jonathan, and M. Leahy, "In vivo imaging of the microcirculation of the volar forearm using correlation mapping optical coherence tomography (cmOCT)," Biomedical Optics Express, vol. 2, no. 5, p. 1184, 2011. 


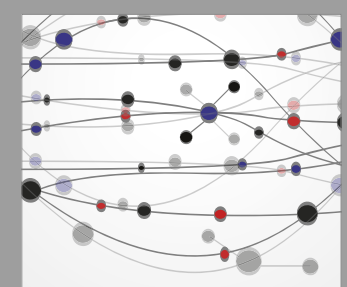

The Scientific World Journal
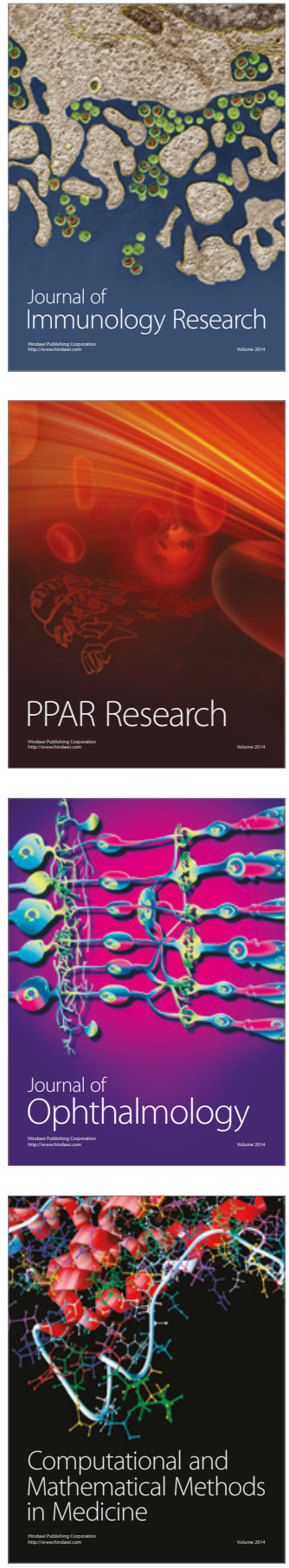

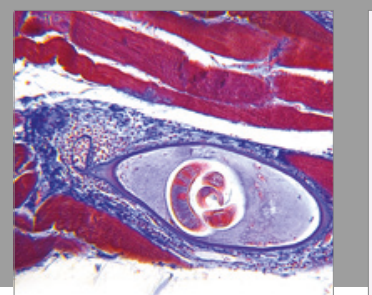

Gastroenterology Research and Practice
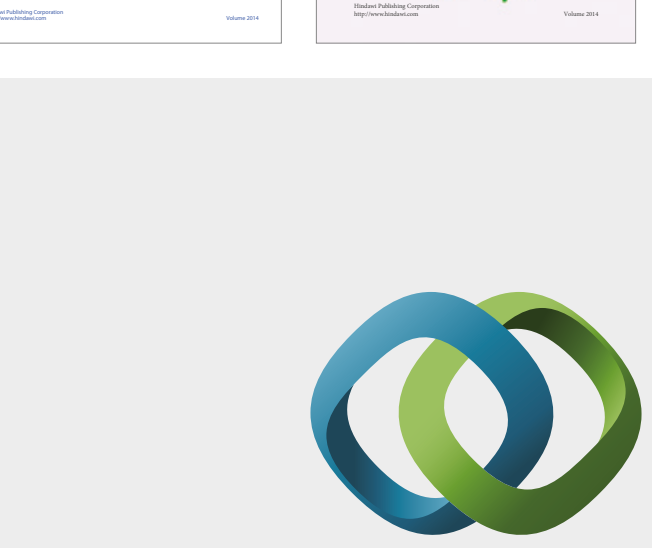

\section{Hindawi}

Submit your manuscripts at

https://www.hindawi.com
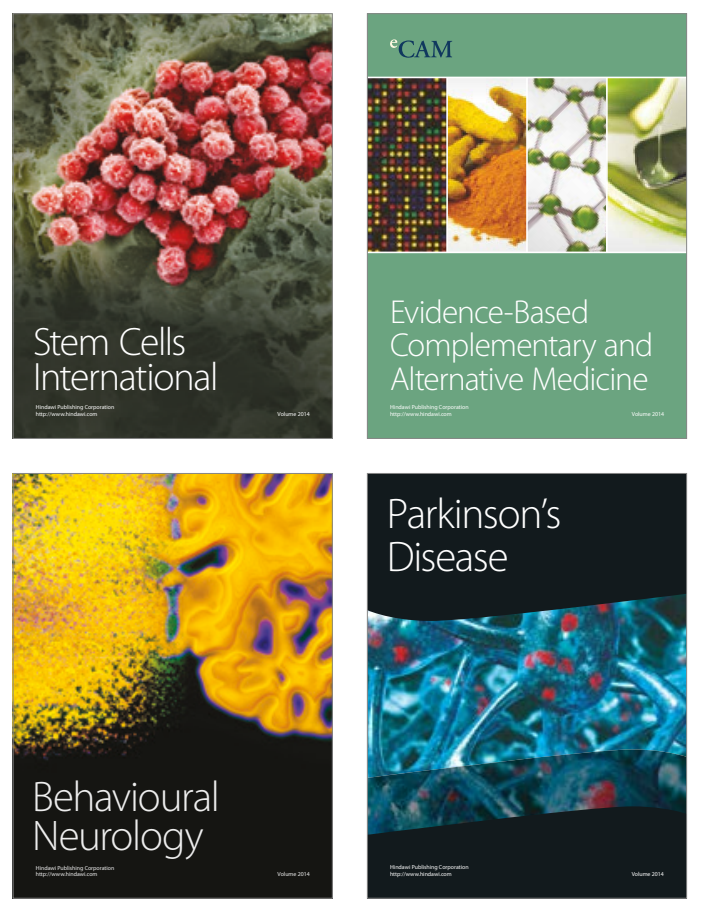
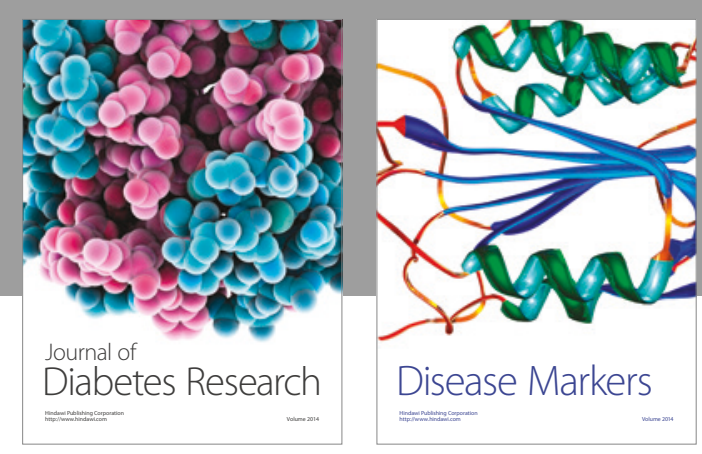

Disease Markers
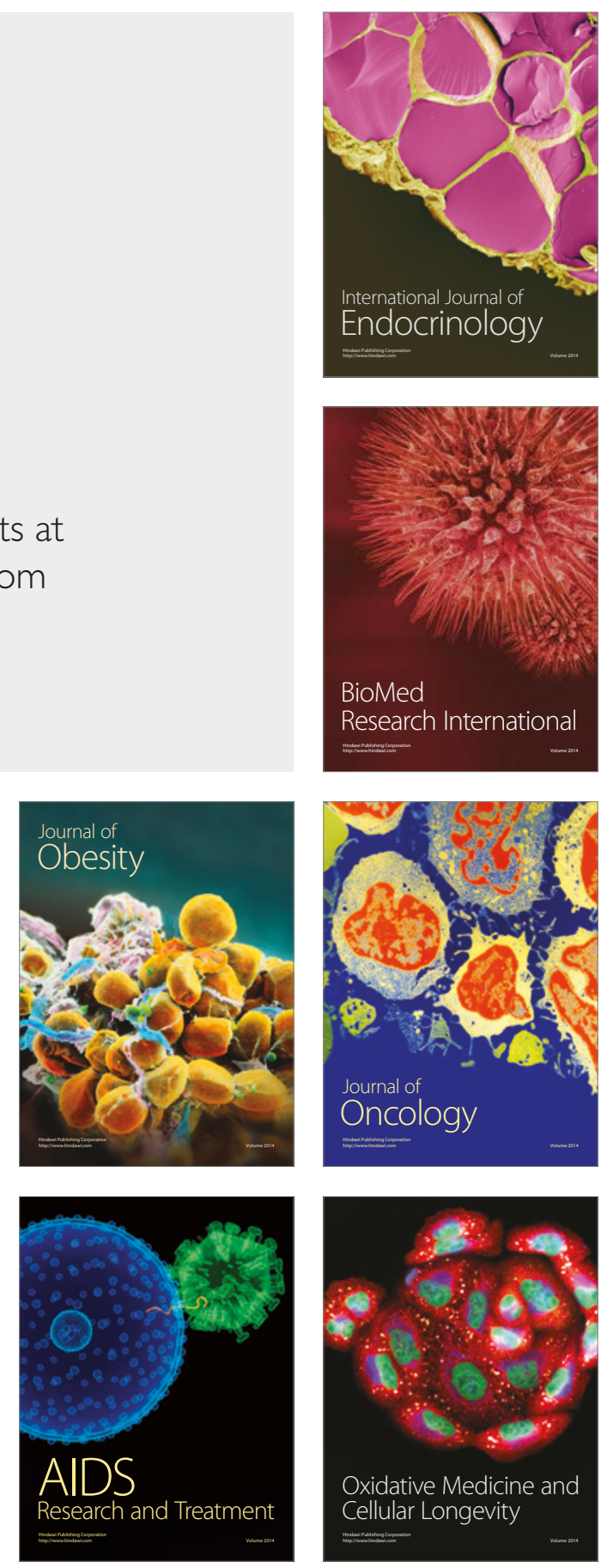